\title{
Bilateral vocal cord paralysis detected incidentally during direct laryngoscopy on general anesthesia
}

\author{
A Ram Doo, Sang-Kyi Lee, and Woo Joo Jeong \\ Department of Anesthesiology and Pain Medicine, Chonbuk National University Medical School, Jeonju, Korea
}

We report a rare case of bilateral vocal cord paralysis detected incidentally during direct laryngoscopy on general endotracheal anesthesia, which had been misdiagnosed as bronchial asthma for over 10 years.

A 20-year-old man, $163.5 \mathrm{~cm}$ in height and $56.2 \mathrm{~kg}$ in weight, was scheduled to undergo septoplasty for sustained snoring and deviation of the external nose. Although the patient had asthma for over 10 years, he presented with no wheezing sounds on the entire lung fields and he had not been treated recently for asthma. Preoperative pulmonary function testing was omitted. About 7 years ago, he had a tonsillectomy and adenoidectomy under general anesthesia uneventfully. General anesthesia was induced with propofol $120 \mathrm{mg}$ and paralysis was facilitated with rocuronium $50 \mathrm{mg}$. Sevoflurane $4 \%$ in $100 \% \mathrm{O}_{2}$ and continuous infusion of remifentanil was started during the mask ventilation. Assuring full muscle paralysis, direct laryngoscopy revealed an extremely narrow glottis gap with bilateral vocal cords which were flaccid but fixed in the paramedian position symmetrically. Subsequently, an additional $10 \mathrm{mg}$ of rocuronium was administered, but the paramedian glottis position was persistent. After a discussion between two different anesthesiologists and one otorhinolaryngologist who also saw the vocal cords in the paramedian position symmetrically under direct laryngoscopy, it was decided that the endotracheal intubation should proceed. A reinforced endotracheal tube of inner-diameter 7.5 $\mathrm{mm}$ was inserted with gentle and forceful insertion pressure and successfully placed into the trachea on the first attempt. Dexamethasone $5 \mathrm{mg}$ was administered intravenously. At the end of surgery, glycopyrrolate $0.4 \mathrm{mg}$ and pyridostigmine $10 \mathrm{mg}$ were given, and the patient was able to open his eyes following a verbal order. After confirming full recovery of the airway reflex and motor functions, the patient was extubated in the operating room. Airway obstruction signs such as stridor, hoarseness, or dyspnea were not observed for 2 hours in the postanesthesia care unit. Oxygen saturation by pulse oximetry was maintained at $100 \%$ on room air. The patient was transferred to the general ward and he recovered without any complication.

The otolaryngologist's laryngoscopic examination was performed on the 3rd postoperative day. The patient presented with bilateral abductor paralysis and the bilateral vocal cords were placed midway between full abduction and adduction on forced inspiration (Fig. 1A). But medial motion, which plays an important role in phonatory vibrations, was relatively preserved, because both vocal folds were in close approximation at rest, which might be advantageous when joining together (Fig. 1B). The patient was interviewed again in detail postoperatively. He had a prior history of asthma for over 10 years, and he had worsening of dyspnea on exertion since high school years. He experienced severe dyspnea three times during military training. Vocal symptoms such as hoarseness, dysphonia and vocal fatigue were not remarkable. Taken together, this may have been a decisive factor in the bilateral vocal cord paralysis being misdiagnosed as bronchial asthma for over 10 years. Vocal symptoms may be more absent in bilateral vocal cord paralysis than in unilateral cases [1].

Occurrence of vocal cord paralysis is associated with a history of thoracic or neck surgery, extralaryngeal malignancy, and various neurologic diseases such as amyotrophic lateral sclerosis and it may also be idiopathic, that is, of unknown origin [2-4]. In this case, although the cause of the vocal cord paralysis was unclear, because of lack of further evaluations, the authors sug-

Corresponding author: Sang-Kyi Lee, M.D., Ph.D., Department of Anesthesiology and Pain Medicine, Chonbuk National University Medical School \& Chonbuk National University Hospital, 20, Gunji-ro, Deokjin-gu, Jeonju 561-712, Korea. Tel: 82-63-250-1250, Fax: 82-63-250-1240, E-mail: leesk@jbnu.ac.kr

(c) This is an open-access article distributed under the terms of the Creative Commons Attribution Non-Commercial License (http:// creativecommons.org/licenses/by-nc/3.0/), which permits unrestricted non-commercial use, distribution, and reproduction in any medium, provided the original work is properly cited. 

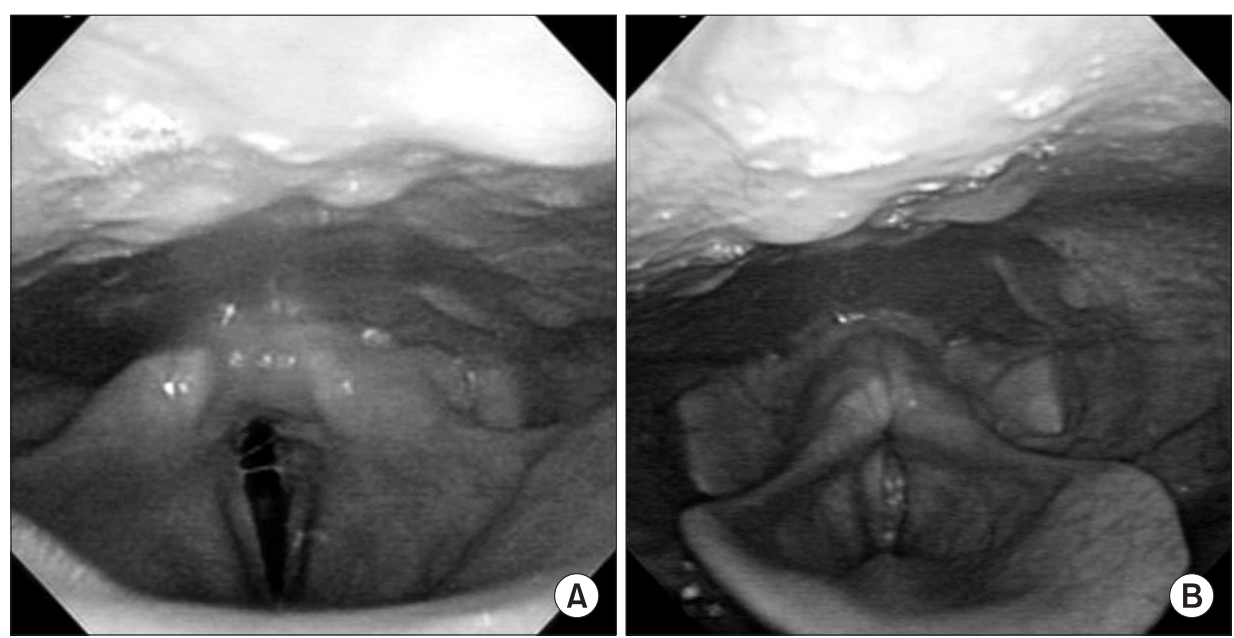

Fig. 1. (A) Larygoscopy showing limited abduction of both vocal folds, with a narrow glottis opening during full inspiration (B) Laryngoscopy during phonation while pronouncing "ee-"

gest that the vocal cord paralysis may have occurred when the patient started suffering from dyspnea over 10 years ago. The paramedian position of the vocal cords was persistent despite the administration of a large dose of rocuronium and this situation is very rare clinically. Muscle relaxants relax the laryngeal muscles including the abductors and adductors, and therefore, the vocal cords become abducted; this is called the cadaveric position [5]. The authors suggest a possible explanation for this is that the patient might have a congenital structural disorder of the larynx and vocal cords with a narrower glottis gap than normal. But further investigations need to be performed including electromyography of the posterior and lateral cricoarytenoid muscles, and that is the limitation of this case.

Vocal cord paralysis of this patient was incidentally detected during direct laryngoscopy on induction of anesthesia. Endotracheal intubation might have possibly failed in this patient because of the narrow glottis opening. Moreover, if the patient's glottis opening became narrower below the critical range of width, because of laryngospasm or laryngeal edema during the induction of the anesthesia, severe airway obstruction may have occurred. Fortunately, air passage through the extremely narrow glottis gap was possible during the mask ventilation and even though the vocal folds were nearly closed, the flaccid both vocal folds could be gently spread apart with the tip of the endotracheal tube, and the patient was intubated by a skilled anesthesiologist. But it is mistake that an endotracheal tube of a smaller size should be selected rather than routine choice for male adults, considering the narrow laryngeal aperture and the possibility of additional laryngeal injuries by a large endotracheal tube. Anesthesiologist should decide whether the patient should be extubated in the operating room or not, and the authors recommend that some means of airway management such as endotracheal reintubation and tracheostomy should be prepared for the possibility of respiratory failure after extubation.

It was the anesthesiologist's mistake not to interview and examine the patient more carefully. If the anesthesiologist had taken the patient's history more carefully and recommended further evaluation such as a pulmonary function test, even though the patient was believed to have had bronchial asthma, this problem could be found preoperatively. The fact that the patient was young and was taking part in military service might interfere with an accurate examination.

In summary, bilateral vocal cord paralysis without significant vocal symptoms may be difficult to detect and may be misdiagnosed as bronchial asthma. Preanesthetic evaluation should be performed vigilantly.

\section{References}

1. Dworkin JP, Treadway C. Idiopathic vocal fold paralysis: clinical course and outcomes. J Neurol Sci 2009; 284: 56-62.

2. Rosenthal LH, Benninger MS, Deeb RH. Vocal fold immobility: a longitudinal analysis of etiology over 20 years. Laryngoscope 2007; 117: 1864-70.

3. Hayward NJ, Grodski S, Yeung M, Johnson WR, Serpell J. Recurrent laryngeal nerve injury in thyroid surgery: a review. ANZ J Surg 2013; 83: $15-21$.

4. Jeong DM, Kim GH, Kim JA, Lee SM. Transient bilateral vocal cord paralysis after endotracheal intubation with double-lumen tube -A case report-. Korean J Anesthesiol 2010; 59 Suppl: S9-12.

5. Krohner RG, Ramanathan S. Functional anatomy of the airway. In: Benumof's Airway Management: Principles and Practice. 2nd ed. Edited by Hagberg CA: Philadelphia, Mosby, Inc. 2007, pp 3-21. 